Pacific Journal of Mathematics

ON ACTION OF SL(2) ON COMPLETE ALGEBRAIC

A. BIAtYNICKI-BIRUL 


\section{ON ACTION OF SL(2) ON COMPLETE} ALGEBRAIC VARIETIES

\section{A. BIALYNICKI-BIRULA}

Dedicated to Gerhard Hochschild on the occasion of his 65th birthday

The aim of this paper is to provide full proofs of results announced in [2]. Some theorems are proved under weaker assumptions. The results lead to a decomposition theorem for actions of SL(2) similar to that proved in [1] for torus actions. However even in this case of $\mathrm{SL}(2)$ and in a greater extent in the case of actions of arbitrary semisimple groups the results are not so full and many questions are left open. Some of them are mentioned in the paper.

All considered algebraic varieties and morphisms are assumed to be defined over an algebraically closed field $k$ (of any characteristic). Let $G$ denote a connected semisimple algebraic group. Let $\alpha: G \times$ $X \rightarrow X$ be an action of $G$ on a complete algebraic variety $X$. For $g \in G$ and $x \in X$ we shall write $g(x)$ or $g x$ instead of $\alpha(g, x)$. The subvariety of fixed points of the action is denoted by $X^{a}$. The orbit $G x$ of $x \in X$ is said to be closed if it is closed in $X$. A closed orbit is said to be nontrivial if it is not composed of one point.

Theorem 1. Let $G, X, \alpha$ be as above. Assume that there exists a dense orbit in $X$. Then $X^{G}$ is finite (but possibly empty) and if the action of $G$ on $X$ is not trivial then $X$ contains a nontrivial closed orbit.

Proof. Let us assume first that $X$ is normal. Then it follows from Lemma 8 of [9] that $X$ can be covered by open quasi-projective $G$-invariant subvarieties. Since $X$ is quasi-compact, $X$ can be covered by a finite number of these and in order to prove that $X^{i}$ is finite it is enough to prove that the result is true when $X$ is quasi-projective. Let $X$ be quasi-projective. Then $X$ can be imbedded in a $G$-invariant way into some projective space $\boldsymbol{P}^{n}$ equipped with a (linear) action of $G$. We are going to fix such an imbedding and consider $X$ as a locally-closed subset of $P^{n}$. Let $a \in X^{a} \subset P^{n}$. It is sufficient to prove that there is an open $G$-invariant neighborhood $U$ of $a$ in $X$ such that $U \cap X^{G}=\{a\}$. It follows from Mumford conjecture (proved by Habush [5]) that there exists a $G$-invariant hypersurface $V \subset P^{n}$ such that $a \notin V$. The closure $\bar{X}$ of $X$ in $P^{n}$ is closed and the difference $\bar{X}-V$ is affine. Since any two closed $G$ invariant orbits in $\bar{X}-V$ have different images in the quotient $(\bar{X}-V) / G$ (see [3]) and since $\bar{X}-V$ contains a dense orbit, we have 
$(X-V)^{a}=\{a\}$. Thus we may take $U=X-V$ and the proof of the first part of the theorem in the case where $X$ is normal is complete.

Now we shall prove the second part of the theorem in the normal case. Assume that the action is not trivial. Let $T \subset X$ be a non-trivial orbit of the smallest dimension. Then $T \cup X^{a}$ is closed and hence complete. Let us assume that $T$ is not closed. Then there exists $a \in \bar{T} \cap X^{G}$. Let $U$ be a quasi-affine $G$-invariant neighborhood of $a$ found as in the first part of the proof. Then $T \subset U$ and hence $T$ is quasi-affine. But the difference $\bar{T}-T$ is a finite subset of $X^{G}$ hence $\operatorname{dim} \bar{T}=\operatorname{dim} T=1$ (if a completion of a quasiaffine variety is finite then the variety is of dimension one). But any one-dimensional orbit of a semisimple group is isomorphic to $P^{1}$ and this gives a contradiction, since we have assumed that the orbit is not closed. Thus the theorem is proved in the normal case.

In the general case it is enough to consider the normalization $\eta: \widetilde{X} \rightarrow X$ of $X$ with the induced action of $G$ on $\widetilde{X}$. Since $\eta\left(\widetilde{X}^{G}\right)=X^{G}$ and $\widetilde{X}^{G}$ is finite (because $X$ is normal and we may apply the theorem in this case), $X^{G}$ is finite. Since $\widetilde{X}$ contains a nontrivial closed orbit, $X$ also contains such an orbit.

COROLlaRY 2. If the action of $G$ on a complete variety $X$ has no nontrivial closed orbit, then the action is trivial.

Theorem 1 shows the importance of closed orbits in the theory of actions of semisimple groups on complete varieties. It suggests that in this theory closed orbits (not only fixed points) play a role analogous to that of fixed points in the theory of actions of multiplicative or additive groups. It can be also noticed here that in the affine case, i.e., if an action of $G$ on an affine veriety $Y$ is given, then $Y$ contains exactly one closed orbit whenever it contains a dense orbit (as follows easily from the Mumford conjecture). In the complete case the analogous result is not valid. Moreover the closure of an orbit may contain an infinite number of closed orbit (see [8], p. 799).

THEOREM 3. Suppose moreover that the variety $X$ is projective and assume that $X$ contains a dense orbit. Then $X^{G}$ contains at most one fixed point.

Proof. Assume that $X$ is normal. Then there exists a $G$ invariant imbedding $X \hookrightarrow P^{n}$, where $n$ is an integer and $P^{n}$ is equipped with a (linear) action of $G$. Let this action be given by $g \rightarrow A(g)$ where $A(g)=\left(a_{i j}(g)\right)$ is a $(n+1) \times(n+1)$ matrix, and 
$i, j=0, \cdots, n$. If $\left[a_{0}, \cdots, a_{n}\right] \in P^{n}$ is a fixed point of the action then, for any $g \in G, A(g)\left[\begin{array}{c}a_{0} \\ \vdots \\ a_{n}\end{array}\right]=\lambda(g)\left[a_{0}, \cdots, a_{n}\right]$ for some $\lambda(g) \in k$ and hence $\lambda(g)=1$, since $G$ has no nontrivial characters. We may assume that the vector subspace of $A^{n+1}$ composed of proper vectors of $A(g)$ for all $g \in G$ is span by $e_{0}=[1,0, \cdots, 0], e_{1}=[0,1,0, \cdots, 0], \cdots$, $e_{k}=[0, \cdots, 0,1,0, \cdots, 0]$. Assume that $\left[a_{0}, \cdots, a_{n}\right]$ and $\left[a_{0}^{\prime}, \cdots, a_{n}^{\prime}\right] \epsilon$ $X^{G} \subset P^{n}$. It follows from the above that $a_{k+1}=\cdots=a_{n}=0$, $a_{k+1}^{\prime}=\cdots=a_{n}^{\prime}=0$. It follows from the Mumford conjecture that there are homogeneous $G$-invariant polynomials $F, F^{\prime} \in k\left[X_{0}, \cdots, X_{n}\right]$ such that $F\left[a_{0}, \cdots, a_{n}\right] \neq 0, F^{\prime}\left(a_{\nu}^{\prime}, \cdots, a_{n}^{\prime}\right) \neq 0$. Of course, we may assume that $\operatorname{deg} F=\operatorname{deg} F^{\prime}$. For $c \in k, F+c F^{\prime}$ is a homogeneous $G$-invariant polynomial and for some $c \in k,\left(F+c F^{\prime}\right)\left(a_{0}, \cdots, a_{n}\right) \neq 0$, $\left(F+a F^{\prime}\right)\left(\alpha_{i}^{\prime}, \cdots, a_{n}^{\prime}\right) \neq 0$. Hence both $\left[a_{0}, \cdots, a_{n}\right]$ and $\left[a_{0}^{\prime}, \cdots, a_{n}^{\prime}\right]$ belong to an open affine $G$-invariant subvariety of $X$. This contradicts existence of exactly one closed orbit in the affine case. Hence Theorem 3 is proved for $X$ normal. If $X$ is not normal then let us consider the normalization $\eta: \widetilde{X} \rightarrow X$ of $X$. Since $\eta\left(\tilde{X}^{G}\right)=X^{G}$, and $\tilde{X}^{a}$ contains at most one point, so does $X^{a}$.

It would be interesting to know if Theorem 3 holds under weaker assumption that $X$ is complete.

Let $X^{*}$ be the union of all closed orbits of the action of $G$ on $X$.

Proposition 4. $X^{*}$ is a closed subset of $X$.

Proof. Let us fixed a Borel subgroup $B \subset G$. If $a \in X$ belongs to a closed orbit, then the isotropy subgroup $G_{a} \subset G$ contains a conjugate of $B$, i.e., the orbit of $a$ contains a point with the isotropy group containing $B$. The set $X^{B}$ is closed hence complete. Let $\beta$ : $G / B \times X^{B} \rightarrow X$ be a map defined by $\beta(g B, a)=g(a)$. It is easy to see that the map is a well defined morphism and it follows from the above that $\beta\left(G / B \times X^{B}\right)=X^{*}$. But since $G / B \times X^{B}$ is complete, its image is also complete hence closed in $X$.

THEOREM 5. $X^{G}$ is a union of some connected components of $X^{*}$.

Proof. Assume that $X$ is normal. Let $a \in X^{a}$. Then there exists an open $G$-invariant quasi-projective neighborhood $U$ of $a$ in $X$ (Lemma 8 in [9]) and there is a G-invariant imbedding $U \hookrightarrow P^{n}$. It follows from the Mumford conjecture that there exists a quasiaffine $G$-invariant open neighborhood of $a$ in $X$. Therefore the only 
closed orbits of $G$ in $U$ are trivial. Hence $X^{G}$ is open in $X^{*}$. Since $X^{G}$ is also closed, the theorem is proved in case where $X$ is normal. In the general case one uses the already proved result for the normalization of $X$.

In the sequel we are going to assume that there exists an open covering $\left\{U_{i}\right\}(i=1, \cdots, k)$ of $X$ such that for each $i$ there exists a $G$-invariant embedding $U_{i} \hookrightarrow P^{n}$, where $n$ is an integer. It is known that if $X$ is normal then it has this property. Let $T$ be a maximal torus of $G$ and let $G_{m} \hookrightarrow T$ be a one-dimensional subtorus satisfying the condition: $X^{T}=X^{G_{m}}$ (see [4] for existence and other pertinent results). Let $X_{G_{m}}^{+}$be the irreducible (and connected) component of $X^{T}$ corresponding to the "big" cell in the decomposition of $X$ determined by the action of $G_{m}$. Let

$$
X_{0}=\bigcup_{G_{m} \rightarrow G} X_{G_{m}}^{+} \text {. }
$$

Theorem 6. Let $G=\mathrm{SL}(2)$. Then $X_{0}$ is a connected and irreducible component of $X^{*}$.

Proof. First, we prove that $X_{G_{m}}^{+} \subset X^{*}$. Let $a \in X_{G_{m}}$. Assume that $a \notin X^{*}$. Then the isotropy group of $a$ is either $G_{m}$ or the normalizer of $G_{m}$ in $G$. The induced action of $G$ on the tangent space $V$ at $a$ to the orbit of $G$ is linear and when diagonalized then it is given by $t\left(x_{1}, x_{2}\right)=\left(t x_{1}, t^{-1} x_{2}\right)$, for $t \in G_{m}$ and $\left(x_{1}, x_{2}\right) \in V$. Hence $a$ does not belong to a "big" cell. Thus $X_{G_{m}}^{+} \subset X^{*}$.

Now, the only parabolic subgroups of $\mathrm{SL}(2)$ are Borel subgroups and SL(2). Hence, there are only two types of closed orbits: fixed points and $\mathrm{SL}(2) / B \approx P^{1}$. Let us assume that the action is nontrivial. Then $X_{0} \cap X^{G}=\varnothing$. (If $a \in X^{G}$ then the induced action of $G$ on the tangent space $T_{a, x}$ is either trivial or the induced action of $G_{m} \hookrightarrow G$ on $T_{a, X}$ contains vectors of both positive and negative weights.) Therefore $X_{0} \subset X^{*}-X^{G}$. Since $X_{0}$ is irreducible, $X_{0}$ is contained in a connected component of $X^{*}$.

Denote the component by $Z$. Fix a torus $G m=T \subset G$ and let $B_{1}, B_{2}$ be the Borel subgroups containing $T$. Then $X_{G_{m}}^{+} \cup Z^{B_{1}} \cup Z^{B_{2}}$ (since the isotopy group of any point from $X_{G_{m}}$ is parabolic and hence contains either $B_{1}$ or $B_{2}$ ). But $X_{\sigma_{m}}^{+}$is connected and $Z^{B_{1}} \cap Z^{B_{2}}=0$ (since $\left.Z^{B_{1}} \cap Z^{B_{2}}=Z^{G}\right)$. Thus either $X_{G_{m}} \subset Z^{B_{1}}$ or $X_{G_{m}}^{+} \subset Z^{B_{2}}$. We may assume that $X_{G_{m}}^{+} \subset Z^{B_{1}}$. But $Z^{R_{1}} \subset Z^{T} \subset X^{T}$, and $X_{G_{m}}^{+}$is connected component of $X^{T}$, hence $X_{G_{m}}^{+}$is a connected component of $Z^{B_{1}}$. Orbit of any point form $Z$ contains a point from $Z^{B_{1}}$. Hence $G Z^{B_{1}}=Z$. Moreover $G / B_{1}$ contains exactly one fixed point for $B_{1}$ and any orbit of a point from $Z$ is isomorphic to $G / B_{1}$. Therefore there exists exactly one con- 
nected component of $Z^{B_{1}}$, i.e., $Z^{B_{1}}$ is connected and we have proved that $Z^{B_{1}}=G_{G_{m}}$. Hence $Z^{B_{1}}$ is irreducible and therefore $Z$ is irreducible. Moreover we have obtained that $X_{0}=G X_{\sigma_{m}}^{+}=Z$ and $X_{0}$ is a connected and irreducible component of $X$.

COROLLARY 7. If the action is not trivial and $\operatorname{ch}(k)=0$, then $X_{0}$ is isomorphic to $X_{G_{m}} \times P^{1}$.

Let $X^{0}$ be the subset of $X$ composed of all points $x \in X$ such that $\overline{G x} \cap X_{0} \neq \varnothing$.

THEOREM 8. $X^{0}$ coinsides with the union of all "big" cells corresponding to actions of maximal subtori induced by the given action of $G$ on $X$. Hence $X^{0}$ is open. In fact $X^{0}$ is the smallest $G$-invariant neighborhood of $X_{0}$.

Proof. Let $U$ be the union of all "big" cells corresponding to actions of maximal subtori. Then $U \subset X^{0}, U$ is open and $G$-invariant. On the other hand, $X^{0}$ is contained in any open $G$-invariant neighborhood of $X_{0}$. Thus $U=X^{0}$ and the theorem is proved.

Corollary 9. If $\operatorname{ch}(k)=0$ and $X_{0}$ is rational, then $X$ is rational. In particular, if the number of closed orbits in $X$ is finite, then $X$ is rational.

Proof. If the action is trivial, then the corollary is also trivial. Suppose that the action is nontrivial. If $X_{0}$ is rational then, $X_{\sigma_{m}}^{+}$is unirational and $X_{G_{m}}^{+} \times P^{1}$ is rational (Corollary 7 ). But $X$ contains $X_{G_{m}}^{+} \times A^{k}$ (where $k$ is a positive integer) as an open subset. Thus $X$ is rational.

Definition 10. Let $G=\operatorname{SL}(2)$ and $X^{*}=X_{1}^{*} \cup \cdots \cup X_{r}^{*}$ be the decomposition of $X^{*}$ into connected components. Let

$$
\left.X_{i}=\{x \in X ; \overline{(G x})_{0} \subset X_{i}^{*}\right\} .
$$

The decomposition $\left\{X_{i}\right\}$ of $X$ will be called the decomposition determined by the action of $G$ on $X$. Subvarieties $X_{i}$, for $i=1, \cdots, r$, will be called cells of the decomposition. Exactly one cell of the decomposition is open in $X$. This cell corresponds to $X_{i}^{*}=X_{0}$ and is equal to $X^{0}$. It will be called the "big" cell of the decomposition.

THEOREM 11. (a) $X=\bigcup_{i=1}^{r} X_{i}, X_{i} \cap X_{j}=\varnothing$ for $i \neq j$.

(b) $X_{i}$ is locally closed.

(c) $X_{i} \supset X_{i}^{*}$ and $X_{\imath}$ is the smallest G-invariant neighborhood 
of $X_{i}^{*}$ in $\bar{X}_{i}$.

Moreover the decomposition $X=\bigcup_{i=1}^{r} X_{i}$ described above is the only decomposition of $X$ satisfying (c).

The theorem follows from Theorem 8 by induction on $\operatorname{dim} X$.

EXAmPle. Assume that $k$ is of characteristic 0. Let an action of $\mathrm{SL}(2)$ on $P^{n}$ be given. Then the action is induced by a linear representation of $\mathrm{SL}(2)$ on $A^{n+1}$ and the representation can be split into a direct sum $V_{0} \oplus V_{1} \oplus \cdots \oplus V_{m}$, where $m$ is an integer and $V_{i}$ (for $i=0, \cdots, m$ ) is a direct sum of irreducible representations of the dominant weight $i$. It is easy to check that in this case $\left(P^{n}\right)^{0}=P^{n}-\operatorname{Proj}\left(V_{0} \oplus \cdots \oplus V_{m-1}\right)$ and that the cells of the decomposition of $P^{n}$ are of the form $\operatorname{Proj}\left(V_{0} \oplus \cdots \oplus V_{i}\right)-\operatorname{Proj}\left(V_{0} \oplus \cdots \oplus V_{i-1}\right)$. Therefore in some sense the decomposition of $X$ described in Definition 10 and Theorem 11 can be considered as a generalization of the splitting of linear representations into a direct sum of sums of isomorphic irreducible representations.

\section{REFERENCES}

1. A. Bialynicki-Birula, Some theorems on actions of algebraic groups, Annals of Math., 98 (1973), 480-497.

2. - On algebraic actions of SL (2), Bull. Acad. Polon. Sci. Ser. math., phys. et astronom, (to appear).

3. M. Demazure, Demonstration de la conjecture de Mumford (d'apres W. Haboush), Seminaire Bourbaki, vol. 1974/75, expose 462, Lecture Notes in Mathematics 514, Springer Verlag.

4. E. Duma, Decompositions of algebraic varieties given by actions of algebraic tori, Bull. Acad. Polon. Sci. Ser. math., phys. et astronom, (to appear).

5. W. Habousch, Reductive groups are geometrically reductive, Annals of Math., 102 (1975), 67-83.

6. T. Kambayashi, Projective representations of algebraic groups of transformations, Amer. J. Math., 88 (1966), 199-205.

7. J. Konarski, Decompositions of normal algebraic varieties determined by an action of a one-dimensional torus, Bull. Acad. Polon. Sci. Ser. math., phys. et astronom, (to appear).

8. W. L. Popov (В. Л. Попоь), Кьазцодномродные аффцнные алгебрацчекце многообразця груллы SL (2), Цзь. Акад. Наук СССР, Серця математцческая 37 (1973), 792-832. 9. H. Sumihiro, Equivariant completion, J. Math. Kyoto Univ., 14 (1974), 1-28.

Received August 14, 1978.

Filtrowa 63/18

02-056 Warsaw, Poland 


\section{PACIFIC JOURNAL OF MATHEMATICS}

\section{EDITORS}

DONALD BABBITT (Managing Editor)

University of Galifornia

Los Angeles, California 90024

HUGo RossI

University of Utah

Salt Lake City, UT 84112

C. C. MOORE AND ANDREW OGG

University of California

Berkeley, CA 94720
J. DUGUNDJI

Department of Mathematics University of Southern California Los Angeles, California 90007

R. FINN AND J. MILGRAM Stanford University Stanford, California 94305

\section{ASSOCIATE EDITORS}

E. F. BECKENBACH

B. H. NEUMANN

F. WOLF

K. YosHIDA

\section{SUPPORTING INSTITUTIONS}

UNIVERSITY OF BRITISH COLUMBIA CALIFORNIA INSTITUTE OF TECHNOLOGY UNIVERSITY OF CALIFORNIA MONTANA STATE UNIVERSITY UNIVERSITY OF NEVADA, RENO NEW MEXICO STATE UNIVERSITY OREGON STATE UNIVERSITY UNIVERSITY OF OREGON
UNIVERSITY OF SOUTHERN CALIFONIA STANFORD UNIVERSITY UNIVERSITY OF HAWAII UNIVERSITY OF TOKYO UNIVERSITY OF UTAH WASHINGTON STATE UNIVERSITY UNIVERSITY OF WASHINGTON 


\section{Pacific Journal of Mathematics}

\section{Vol. 86, No. $1 \quad$ November, 1980}

Gert Einar Torsten Almkvist, Invariants, mostly old ones .............. 1

Hyman Bass, Groups of integral representation type ................ 15

A. Białynicki-Birula, On action of SL(2) on complete algebraic

varieties........................................

Frederick Paul Greenleaf and Martin Allen Moskowitz, Groups of

automorphisms of Lie groups: density properties, bounded orbits, and

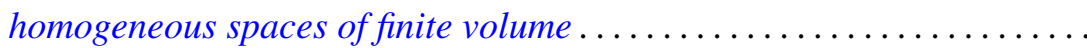

Raymond Taylor Hoobler, A cohomological interpretation of Brauer groups

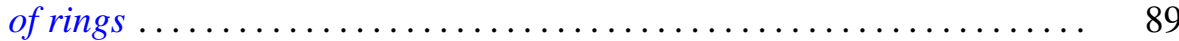

Irving Kaplansky, Superalgebras ........................ 93

Jerrold Lewis Kleinstein and Alex I. Rosenberg, Succinct and

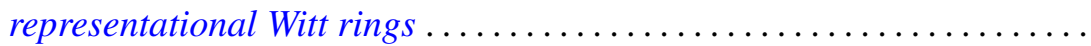

E. R. Kolchin, On universal extensions of differential fields ............ 139

Andy R. Magid, Analytic subgroups of affine algebraic groups. II ....... 145

Calvin Cooper Moore, The Mautner phenomenon for general unitary

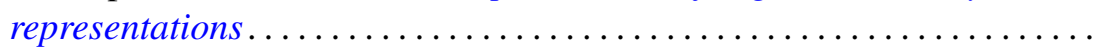

George Daniel Mostow, On a remarkable class of polyhedra in complex hyperbolic space ................................ 171

Brian Lee Peterson, Extensions of pro-affine algebraic groups. II . . . . . . 277

John Henry Reinoehl, Lie algebras and affine algebraic groups......... 287

Maxwell Alexander Rosenlicht, Differential valuations .

John Brendan Sullivan, The second Lie algebra cohomology group and Weyl modules..................................... 321

Moss Eisenberg Sweedler, Right derivations and right differential

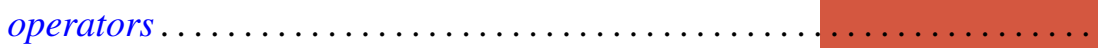

Bostwick Frampton Wyman, Time varying linear discrete-time systems. II. Duality.................................. 